\title{
A Method to Implement Scheduling and Prioritizing of Messages in VANET's
}

\author{
K Jyostna, K Navaneetha
}

\begin{abstract}
Vehicular Adhoc Network (VANET) could be a technology that focuses on increasing convenience and safety of passengers and drivers. Vehicular Adhoc Network's applications will suffer from severe performance degradation in high density state of affairs because of channel congestion.Congestion management ought to be taken into consideration to improvise safety, performance \& dependability of VANETs. during this paper, we have a tendency to projected a technique DySch that assign the priorities to service and safety messages supported the message content (static factor), message size and Dynamic Factor(network state). In DySch strategy, messages is scheduled severally, heuristically and dynamically. The investigation of performance is done by mistreatment urban and main road situations whereas the packet ratio, average delay, average output, and queuing delay are thought-about. as compared to the simplest standard methods, DySch strategy Simulation results shows that the performance of VANETs is improved. to manage congestion in VANETs, the projected strategy will help in improvising safety \& dependability by assigning a priority as higher to the security messages.
\end{abstract}

\section{Index Terms-VANET, Throughput, Packet Loss Ratio.}

\section{INTRODUCTION}

Vehicular impromptu network may be a network of vehicles moving at comparatively high speed, that communicate among themselves with completely different functions. Dedicated Short vary Communication (DSRC) is employed to outline standards and protocols for the communication among vehicles in Intelligent facility. VANETs are completely different from different networks as they need special characteristics like high amendment in topology rate, high quality of nodes, high node density, sharing the wireless channel, and often broken path. But, these special characteristics in VANET create to some issues in information transfer and scheduling [1]-[4]. Congestion happens within the network once the channels are saturated thanks to the high density of nodes. In different words, once the vehicles send messages at the same time when a bigger range of vehicles are transferring, the shared channels get full simply, that ends up in overladen MAC channels, and additionally ends up in increase in average delay and loss of packets, and consequently decreases the VANET performance. Therefore, dominant the congestion enhances the reliableness of VANET [5]-[8].

DSRC uses a information measure of $75 \mathrm{MHz}$ at 5.9 gigahertz for transferring the protection and repair messages

K Jyostna, Department of Electronics and Communication Engineering,VNR Vignana Jyothi Institute of Engineering and Technology, Hyderabad, Telangana, India.(Email: jyostna_k@vnrvjiet.in)

K Navaneetha, Department of Electronics and Communication Engineering, VNR Vignana Jyothi Institute of Engineering and Technology, Hyderabad, Telangana, India.(Email: k.navaneetha421@gmail.com).
Revised Manuscript Received on September 14, 2019.

and performing arts communications in between $\mathrm{V} 2 \mathrm{~V}$ and V2I. To manage the network performance by Wireless Access in transport setting (WAVE) systems DSRC employs IEEE 1609 and IEEE 802.11p standards. Multichannel in VANET is enforced by exploitation IEEE 1609.4 standard. The DSRC information measure consists of channels(eight) which are composed of $10 \mathrm{MHz}$ (six) service channels $(\mathrm{SCH})$ for service-based communications, for safety communications $10 \mathrm{MHz}$ (one) management channel $(\mathrm{CCH})$, and one channel of $5 \mathrm{MHz}$ is used for future use.

Fig. 1 shows allocation of channels at intervals DSRC. The Messages priorities are assigned by exploitation service and management communication channels.

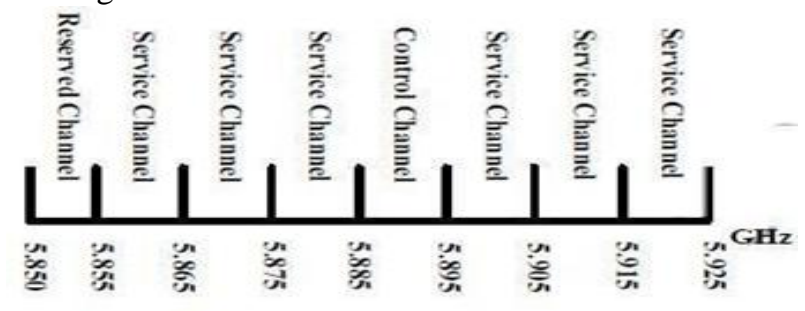

Fig 1: DSRC Channel Allocation

A congestion management strategy that is given during this paper is employed to range and schedule the security and repair messages. The planned strategy consists of priority assignment unit and message planning/scheduling unit.Priority is allotted to any or all the messages supported dynamic and static factors by victimization the priority assignment unit and prioritized messages are rescheduled within the management and repair queues of channels by using the message planning/scheduling unit. the assorted performance parameters like packet ratio, average delay and out turn are wont to appraise the performance of the planned strategy.

\section{LITERATURE SURVEY}

Congestion management algorithms are used to ascertain extremely reliable communication and economical use of information measure within the network. These are usually classified into two sorts of management ways 1) Open loopconsideration of congestion before occurence.2)closed loop - consideration of congestion after occurrence VANET's congestion management ways are classified into three types:

1. The facility of transmissions controlling

2.The speed of transmissions controlling, and

3 Scheduling and Prioritizing messages within the channels. 
The third one is that the commonest in open loop system.

FIFO is one in every of the simplest and essential programing algorithms or scheduling algorithms enforced, the fastest arrived is that the 1 st to be delivered.(MQIF) considers the staleness of information and responsivenes severally schedules the messages supported (QoD) and (QoS)factors . the chance will be given to the data( messages) that had least opportunity is to be served before in Least selected first (LSF) formula The Priorities of messages are outlined finally in $D * S$ algorithm supported point (D) and length of message(S).

All the higher than congestion management ways show deficiency after they are applied into follow.The emergency messages are given sufficient attention by some congestion management algorithms, the more delay, or the more packet ratio for urgent messages cause unreliable and unsafe things in VANETs. It may be determined that there will be a rise in congestion price and reduce in output. In these ways, CSMA/CA protocol is employed to access communication by using the mechanism of backoff-exponential[10]. This mechanism isn't economical for broadcasting the beacon messages in denser network, the messages are going to be timed-out and a rise in born packets may be determined before transmission.

\section{PROPOSED SYSTEM}

IEEE 1609.4 WAVE that allows many channels communications in VANET prioritizes and schedules varied messages. Prioritizing and programing/scheduling the messages are crucial tasks in VANET as sizable amount of metrics ought to be thought of, especially in giant networks. to improvise safety and reliableness of VANETs a congestion management strategy is projected during this paper by using more practical programing/scheduling and prioritizing algorithms

To assign the priority and schedule the messages within the management and repair channels, several factors associated with messages content and physical properties of vehicles are thought of like kind and validity of messages,size of messages, speed of receivers and senders, and more. Fig. 2 shows the layout of the projected congestion management methods. Two units are thought of during this strategy : A) Assignment of priority unit and B) Programing of message unit. The priority assignment unit takes care of distribution priority to any or all the messages supported static and dynamic factors. within the management and repair channels the rescheduling of the prioritized messages in keeping with their properties is completed by message programing unit These are distributed methods as a result of every node in the network severally schedules and prioritizes the messages. The projected congestion management strategy is open-loop strategy that prioritizes and schedules messages by avoiding congestion prevalence.

\section{A. Priority Assignment Unit}

The priorities are appointed to the messages in vehicles,generated by applications in priority assignment unit .Based on the appointed priorities the transmission variable times are determined for the messages. The priority

of every message here is appointed supported dynamic and static factors still as size of message.

$$
\text { Priority }_{\text {Message }}=\frac{\text { Static }_{\text {Factor }} \times \text { Dynamic }_{\text {Faactor }}}{\text { Message }_{\text {Size }}}
$$

1. PriorityService-Low is appointed to the messages derived by priority service applications as low service like parking spot locater, instant electronic communication (between vehicles),internet service provisioning,electronic toll payment, and more.

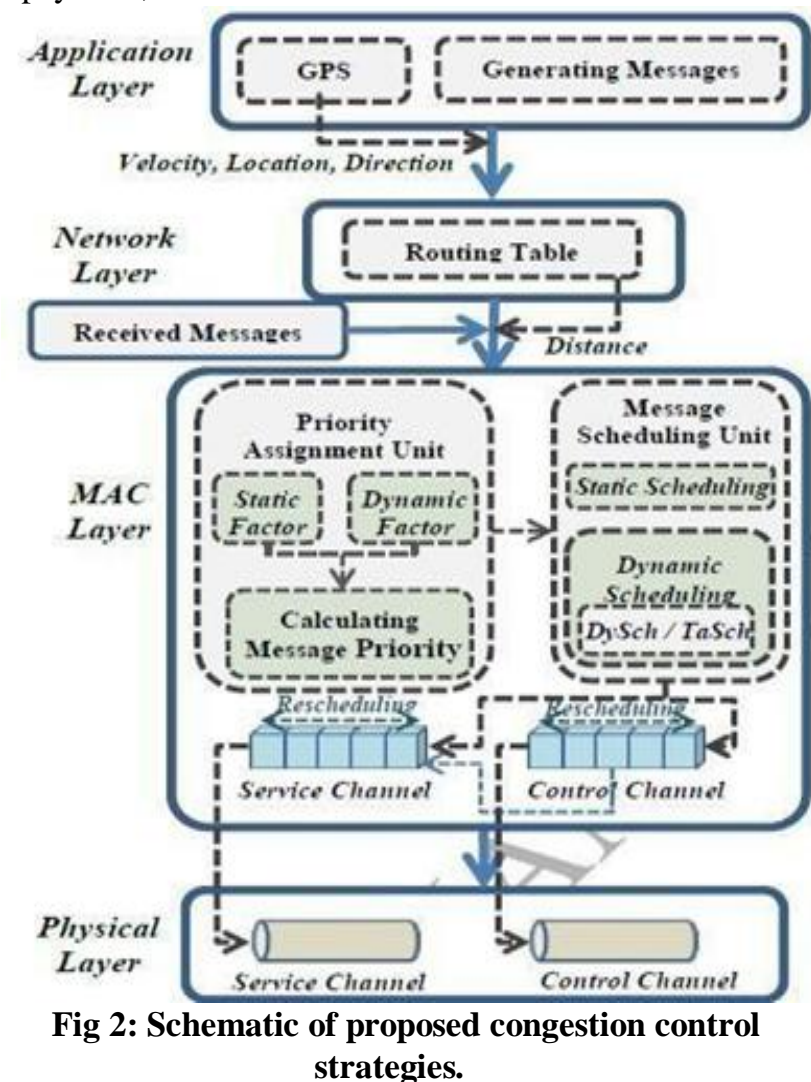

PriorityService-High is allotted to the messages that are derived from service applications which has priority high like intelligent flow of traffic management and map download/update/GPS correction and a lot of.

3.PrioritySafety-Low is taken into account for low priority safety messages generated by the applications of forward collision, lane modification warning, left flip assist,stop sign assist, so a lot of.

4.The beacon safety messages that are sporadically sent in VANETs that used for broadcast in the conveyance data like position, speed, direction, so on are thought-about by Priority Beacon .For many of the protection applications and a few of the applications(service) this data is very important

5.For the urgent messages Priority Emergency is taken into account. These type of messages will have the best priorities \& might delivered with none delay. a number of the applications that will generate emergency messages are emergency vehicle approaching warning,urgent brake lights, crosswalk data, urgent vehicle at scene warning,collision warning intersection, and more. 


\section{B. Message Scheduling}

The message Scheduling is crucial to supply a reliable information transferring. However, it's a difficult task in VANETs as a result of its distinctive characteristics.In order to boost safety and dependableness in VANET's, IEEE 1609.4 many channel waterproof is improved during this paper. The management and repair queues of channels are rescheduled in preference to channels transferring within the message scheduling unit. For this reason, the scheduling of message is conducted in 2steps of static and dynamic scheduling. within the static planning state,the messages will be transferred to either service channel queue or management channel queue supported static issue outlined in assignment of priority unit. because of 2 styles of channels (control and service) in VANETs ,two channel queues (control and service) were assumed . In static planning step, the reports/messgaes with Priority Service-High and Priorities of Priority Service-Low are transferred to service queue channel and therefore the messages with Priority Emergency, Priority Beacon, and Priorities of Priority Safety-Low are transferred to manage queue channel. additionally, once the management queue channel is filled, the Priority Beacon messages ,Priority Safety-Low priorities, and Priority Emergency are pushed to service queue channel for rising protection in VANETs. Fig. 3 is the message planning unit victimisation static scheduling method

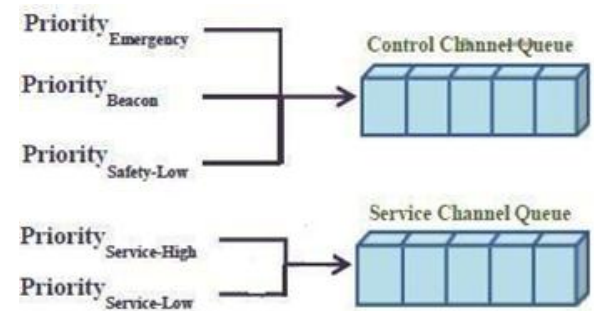

Fig 3: Static scheduling process.

The scheduling(dynamic) state is allotted in 2 totally dissimilar methods: i) exploitation the priorities of mssages calculated in assignment of priority unit, and ii) exploitation the techniques of meta-heuristic for message rescheduling in every queue. For scheduling(dynamic) supported the priorities of message (i), once a replacement packet is burst in to the queue, the data packets in every queue are rescheduled. Indeed, the data packets in every queue are counted degressive supported the priorities are calculated by (1). The packets are removed from queue from the management or service queue channels to transfer to the service or control channels. "DySch", is that the method used for programming(dynamic) .Indeed, DySch is employed to point a method that simply uses outlined priorities for scheduling the management and repair dynamic scheduling queues for .

The techniques of meta-heuristic may be extensively utilized for dynamic scheduling. Reviewing that the easy scheduling issues are NP-hard [6], the scheduling of message is additionally AN NP-hard drawback because of the limitations of the conveyance domains.

\section{SIMULATION RESULTS}

In this paper, the concept of scheduling and giving high priority to emergency messages in VANET application is discussed. EDCA technique is already available to give high priority to emergency messages. But this technique will have high delay as number of vehicles increase and it doesn't consider static and dynamic factor, which helps in efficient calculation of priority messages.NS-2 is the software used for simulating this scenario.

The static factor used in assigning priorities is in ranges of 1 to $5(1,2,3,4$, are consider as lower priority and 5 consider as high priority). Dynamic factors considered are size of message content and distance between vehicles.

Formula to calculate priority using DYSCH technique is Priority $=$ static_factor $*$ dynamic_factor $/$ message_size; For example, (calculate for packet 1)

Static_factor $=3$; (as low priority) Dynamic_factor $($ distance $)=600$ Meters Message_size $=5$;

Priority $=3 * 600 / 5=360$ (as lower priority) Another example (calculate for packet 2) Static_factor $=5$; (as high priority) Dynamic_factor (distance) = 300 Meters Message_size $=3$;

Priority $=5 * 300 / 5=500$ (500 is higher priority compare to 360 . Thus, packet 2 will be scheduled)

\section{Enter below command}

\section{\$ ns EDCA.tcl}

In below screen we can see scheduling of messages at different time without considering static and dynamic factor.
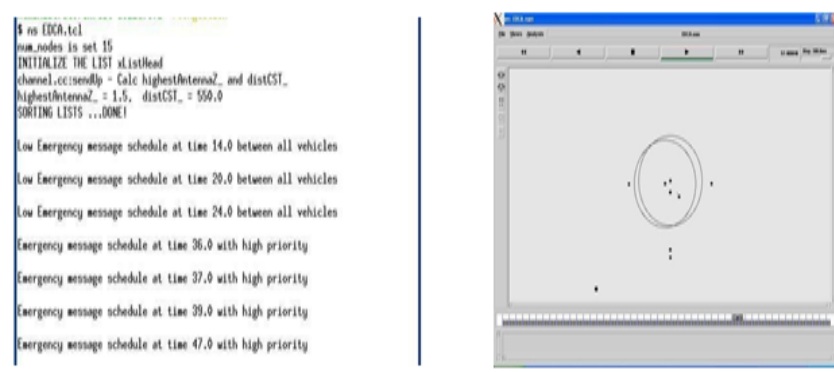

\section{Simulation screen for EDCA}

Enter below command

\$ns DYSCH.tcl

DYSCH technique can be simulated similarly but internally it will consider priority factor calculation. Now run DYSCH.tcl
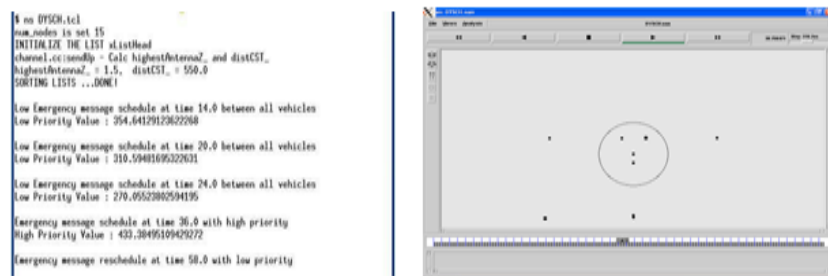

Simulation screen for DYSCH

Published By:

Blue Eyes Intelligence Engineering

\& Sciences Publication 
From the above figure, we can observe that message from high priority vehicle is considered as emergency and it is scheduled first for transmission.

Run below command to get dropped packet information from both techniques

\$ awk -f drop.awk EDCA.tr

.\$ awk -f drop.awk DYSCH.tr

Get information below after running above command

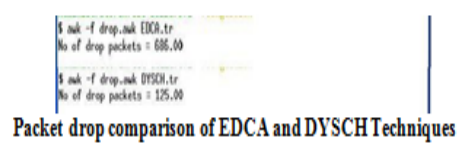

In above screen we can see EDCA drops are 686 and DYSCH drops are 125.

Now calculate Packet Delivery Ratio(PDR) using below command

Sawk-f pdr.awkEDCA.tr

S awk-f pdrawkDYSCH.tr

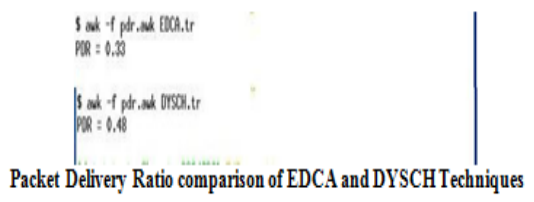

In above screen, $\mathrm{PDR}$ of EDCA is less than DYSCH

Now run delay metric

S awk-f delay.awk EDCA.tr

S awk-f delay.awk DYSCH.tr
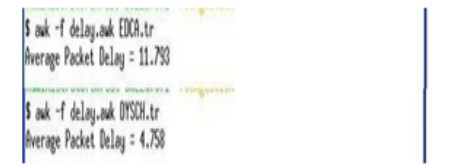

Packet delay comparison of EDCA and DYSCH Techniques

We can observe that EDCA delay is $11.793 \mathrm{msec}$ per packet and DYSCH is $4.758 \mathrm{msec}$ delay

Now run Throughput metric using command

Sawk-f EDCA throughput.awkEDCA.tr

Sawk-f EDCA_throughput.awk DYSCH.tr
\$ auk -f DYSCH_throughput awk DYSCH.tr
Average Throughput[kbps] $=162.38$
\$ auk - $f$ EDCA_throughput.auk EDCA.tı Average Throughput[kbps] $=100.84$
In the below figure it is observed that EDCA throughput is lesser than DYSCH and the throughput graph is generated using the command

.\$xgraphEDCA_throughput.xgr DYSCH_throughput.xgr

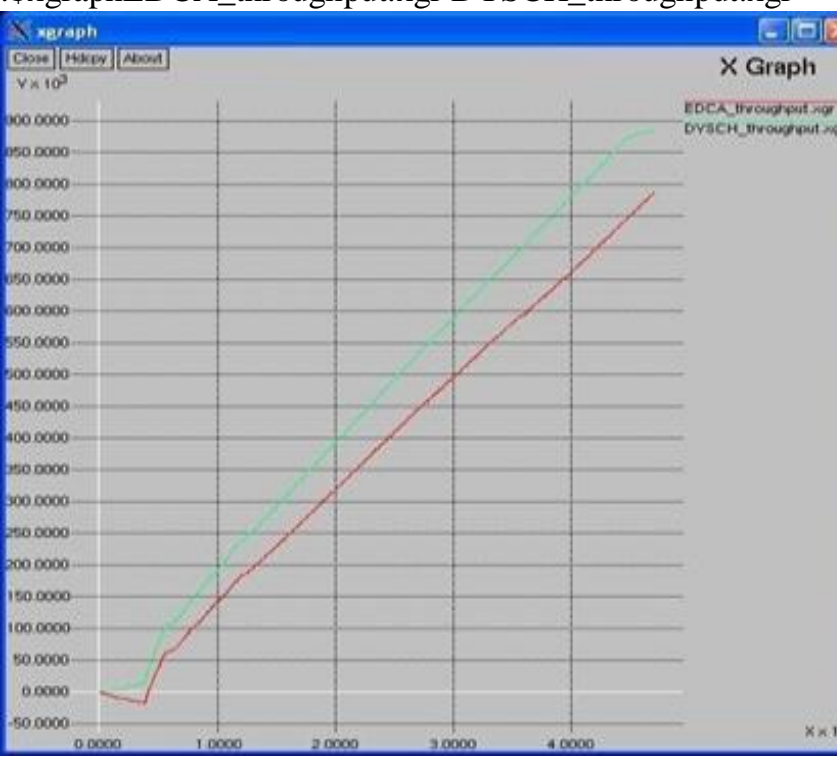
Techniques as Graph
Throughput comparison of EDCA and DYSCH

\section{CONCLUSION}

Two novel open-loop congestion management ways as well as DySch strategy are planned during this paper. The planned ways operated through assignment of priority unit and message planning/scheduling unit. Dynamic and Static factors are calculated supported the messages contains 1 st in priority assignment unit, and state of affairs of automobiles, severally. A priority was assigned then to every message supported dynamic and static factors, and message size. The messages are sent to regulate and repair queue channels supported the deliberated static factors (static planning) so the packets in each queue will be rescheduled for sending to the channels scheduling (dynamic) in message scheduling unit, Dynamic planning was meted out supported the priority of messages in DySch strategy. DySch could be a distributed strategy during which every vehicle is severally prioritized and regular..

The performance of DySch strategy was evaluated and compared with EDCA strategy in urban situation. The results showed that the planned strategy outperforms the EDCA technique. The performance of VANETs is improved by Application of DySch strategy, increasing the typical outturn, and reducing the quantity of loss of packets and delay.The results showed that the applications of DySch raise to the lesser delay in queue waiting, and packet ratio for urgent messages instead of the messages in service channel. Therefore, additional reliable and safe domains are often give in VANETs exploitation DySch strategy.

\section{REFERENCES}

1. F. Sattar,A. Jundi,K. Golestan, , F. Karray L. Nassar, "Vehicular ad-hoc networks (VANETs): challenges,capabilities, in operation and knowledge fusion," Intelligent and Autonomous Systems, ed: Springer, pp. 34-41, 2012.

2. J. Guerrero-Ibáñez, and S. Zeadally, "Vehicular Ad-hoc Networks (VANETs):protocols, design, and Applications,"Wireless Technologies next generation, Springer, pp. 49-70, 2013.

3. G. Karagiannis, E. Ekici,B. Jarupan, and T. Weil, "Vehicular networking: A tutorial and survey on architectiures, challenges, solutions and standards," Communications Tutorials \& Surveys, IEEE, vol. 13, pp. 584-616, 2011

4. S. Zeadally, Y.-S. Chen, and A. Hassan, "Vehicular unintended networks (VANETS): results, and challenges," Telecommunication Systems, vol. 50, pp. 217-241, 2012.

5. S. Mitra, "Management of Congestion by dynamic sharing of data measure among vehicles in VANET," twelfth International Conference on Intelligent Systems style and Applications (ISDA), pp. 291-296, 2012.

6. M. Jabbarpour Sattari "D-FPAV congestion management for VANETS for algorithmic rule to rescue human lives," Archives Des Science, vol. 65, 2012.

7. J. Mittag, H. Hartenstein, "Awareness and congestion management in cooperative transport systems," Proceedings of the IEEE, vol. 99, pp. 1260-1279, 2011.

8. R. K. Singh and "Routing challenges In unintended Networks: A Survey," IJECCE, vol. 3, pp. 126- 132, 2012. 
9. L. Stibor, H.-J. Reumerman, and A. Barroso, "management og congestion in wireless networks for safety transport applications," Proceedings of the eighth European Wireless Conference, 2007.

10. R. M. Noor "A taxonomy for management of congestion algorithms in uintended transport networks," IEEE International Conference on Communication Networks and Satellite (ComNetSat), pp. 44-49,2012,.

11. B. B. Dubey, N. Chand et al., Wireless Networks, vol. 22, no. 5, pp. 1641-1645, 2016.

12. NasrinTaherkhani,Prioritizing of Messages for Congestion management in unintended transport Networks, pc Networks (2016).

13. Ajay Guleria,Narottam chand, QOS Aware service programming theme for VANET's ,2015. 